\title{
Electrically Tunable Plasmonic Resonances with Graphene
}

Emani, Naresh K.; Chung, Ting-Fung; Ni, Xingjie; Kildishev, Alexander; Chen, Yong P.; Boltasseva, Alexandra

Published in:

CLEO Technical Digest

Publication date:

2012

Document Version

Publisher's PDF, also known as Version of record

Link back to DTU Orbit

Citation (APA):

Emani, N. K., Chung, T-F., Ni, X., Kildishev, A., Chen, Y. P., \& Boltasseva, A. (2012). Electrically Tunable Plasmonic Resonances with Graphene. In CLEO Technical Digest (pp. JTu1M.2). Optical Society of America.

\section{General rights}

Copyright and moral rights for the publications made accessible in the public portal are retained by the authors and/or other copyright owners and it is a condition of accessing publications that users recognise and abide by the legal requirements associated with these rights.

- Users may download and print one copy of any publication from the public portal for the purpose of private study or research.

- You may not further distribute the material or use it for any profit-making activity or commercial gain

- You may freely distribute the URL identifying the publication in the public portal

If you believe that this document breaches copyright please contact us providing details, and we will remove access to the work immediately and investigate your claim 


\title{
Electrically Tunable Plasmonic Resonances with Graphene
}

\author{
Naresh K. Emani ${ }^{1}$, Ting-Fung Chung ${ }^{2}$, Xingjie Ni ${ }^{1}$, Alexander Kildishev ${ }^{1}$, Yong P. Chen ${ }^{2}$, \\ and Alexandra Boltasseva ${ }^{*, 1,3,4}$ \\ ${ }^{1}$ School of Electrical and Computer Engineering and Birck Nanotechnology Center, Purdue University, West Lafayette, IN 47907, USA \\ ${ }^{2}$ Department of Physics and Birck Nanotechnology Center, Purdue University, West Lafayette, IN 47907, USA \\ ${ }^{3}$ DTU Fotonik, Department of Photonics Engineering, Technical University of Denmark, Lyngby, DK-2800, Denmark \\ ${ }^{4}$ Erlangen Graduate School of Advanced Optical Technologies (SAOT), Universität Erlangen-Nürnberg, Paul-Gordan-Str. 6, 91052 Erlangen, \\ Germany \\ Email: aeb@purdue.edu ${ }^{*}$
}

\begin{abstract}
Real time switching of a plasmonic resonance may find numerous applications in subwavelength optoelectronics, spectroscopy and sensing. We take advantage of electrically tunable interband transitions in graphene to control the strength of the plasmonic resonance.

OCIS codes: (250.5403) Plasmonics; (160.4670) Optical materials; (160.3918) Metamaterials
\end{abstract}

\section{Introduction}

Plasmonics offers an exciting route to subwavelength optoelectronics by confining the optical fields below the diffraction limit [1]. This is achieved by employing metal nanostructures resonant at a particular optical wavelength. Dynamic control of these plasmonic resonances is critical for many applications like integrated modulators and switches. Active electrical control of resonances has been demonstrated at $\mathrm{THz}$ frequencies [2]. Real time switching of plasmonic resonances at optical wavelengths could open up many exciting possibilities. In this paper we demonstrate voltage controlled plasmonic resonance using graphene. Graphene is a hexagonal lattice of $\mathrm{sp}^{2}$ carbon atoms in a single sheet with extraordinary electrical and optical properties [ $[3,4]$, that was proposed as a platform for integrated optoelectronics and transformation optics [5-7]. Strong graphene-based modulation has recently been demonstrated for on-chip applications []].

\section{Tunable Plasmonic Resonances}

Graphene exhibits a unique linear dispersion relation shown in Fig. 1a. The carrier density $\left(\mathrm{n}_{2 \mathrm{D}}\right)$ can be electrically controlled by an applied gate voltage, leading hereby to voltage controlled Fermi energy $\mathrm{E}_{\mathrm{F}}$. A direct consequence of that is the gate tunable interband threshold, wherein an incident photon can generate an electron-hole (e-h) pair only if its energy is higher than $2 \mathrm{E}_{\mathrm{F}}$. For photons of lower energy there will not be interband losses at $0 \mathrm{~K}$. At higher temperatures the interband threshold is broadened but shows qualitatively similar behavior. For realistic carrier densities in the range $10^{11}-10^{13} \mathrm{~cm}^{-2}$ the interband threshold can be continuously tuned up to near-infrared (NIR) frequencies. When metal nanostructures with resonant frequency in the NIR are fabricated on top of graphene sheet their optical properties can be tuned by the applied gate voltage. The resonance is also accompanied by a local field enhancement which causes interaction with the graphene sheet. Hence, if the resonant frequency is above the interband threshold then strong interband losses are present and the resonance is considerably weakened.

Fig. $2 b$ shows a schematic of the experimental setup for demonstrating plasmon resonance tuning. The source-drain contacts were deposited by e-beam evaporation of $10 \mathrm{~nm} \mathrm{Ti}$ and $50 \mathrm{~nm} \mathrm{Au}$ on doped Si substrate with $100 \mathrm{~nm}$ silicon oxide. A large area graphene sheet grown by chemical vapor deposition (CVD) was transferred onto the substrate ensuring electrical contact with both source and drain. Then, bow-tie like plasmonic structures were fabricated by electron beam lithography, metallization $(2 \mathrm{~nm} \mathrm{Ti}$ and $30 \mathrm{~nm} \mathrm{Au})$ and subsequent lift-off processes. The gate voltage applied to the Si substrate controls the carrier concentration in graphene sheet because of the field effect. The resistance between source and drain contacts was monitored to verify the field effect. A Fourier Transform Infrared (FTIR) spectrometer was used to optically characterize the plasmonic resonance behavior. A scanning electron micrograph of the fabricated sample with plasmonic structures on top of the graphene sheet is shown in Fig. 1c. It is clearly seen that CVD graphene is mostly single layered (verified separately by Raman spectroscopy) over the area of $\sim 16 \mathrm{~mm}^{2}$. The optical transmission data shows a resonance at $\sim 3 \mu \mathrm{m}$ that is in agreement with the simulated data for the chosen resonator design. The variation of the source-drain resistance with gate voltage is shown in Fig. 1d. Graphene shows minimum carrier concentration at a voltage of $8 \mathrm{~V}$ that is referred as the Dirac point in literature. At voltages lower than the Dirac point, the hole concentration increases progressively moving the interband threshold towards NIR frequencies. Fig. 1e shows the initial results demonstrating weakening of the resonance with increasing carrier concentration. The percentage change in the resonance strength with applied 
voltage is up to $6 \%$ in the current sample as shown in Fig. 1f. We note that the maximum change in resonance occurs at $3.6 \mu \mathrm{m}$ and can be further improved with higher carrier concentration. Further, the observed change of $6 \%$ is seen off-resonance and we believe that the change would be much higher with plasmon resonance at $4.5 \mu \mathrm{m}$ and improved fabrication processes. In summary, we have demonstrated electrically controlled plasmonic resonance using graphene, and efforts are underway to achieve even higher tunability.
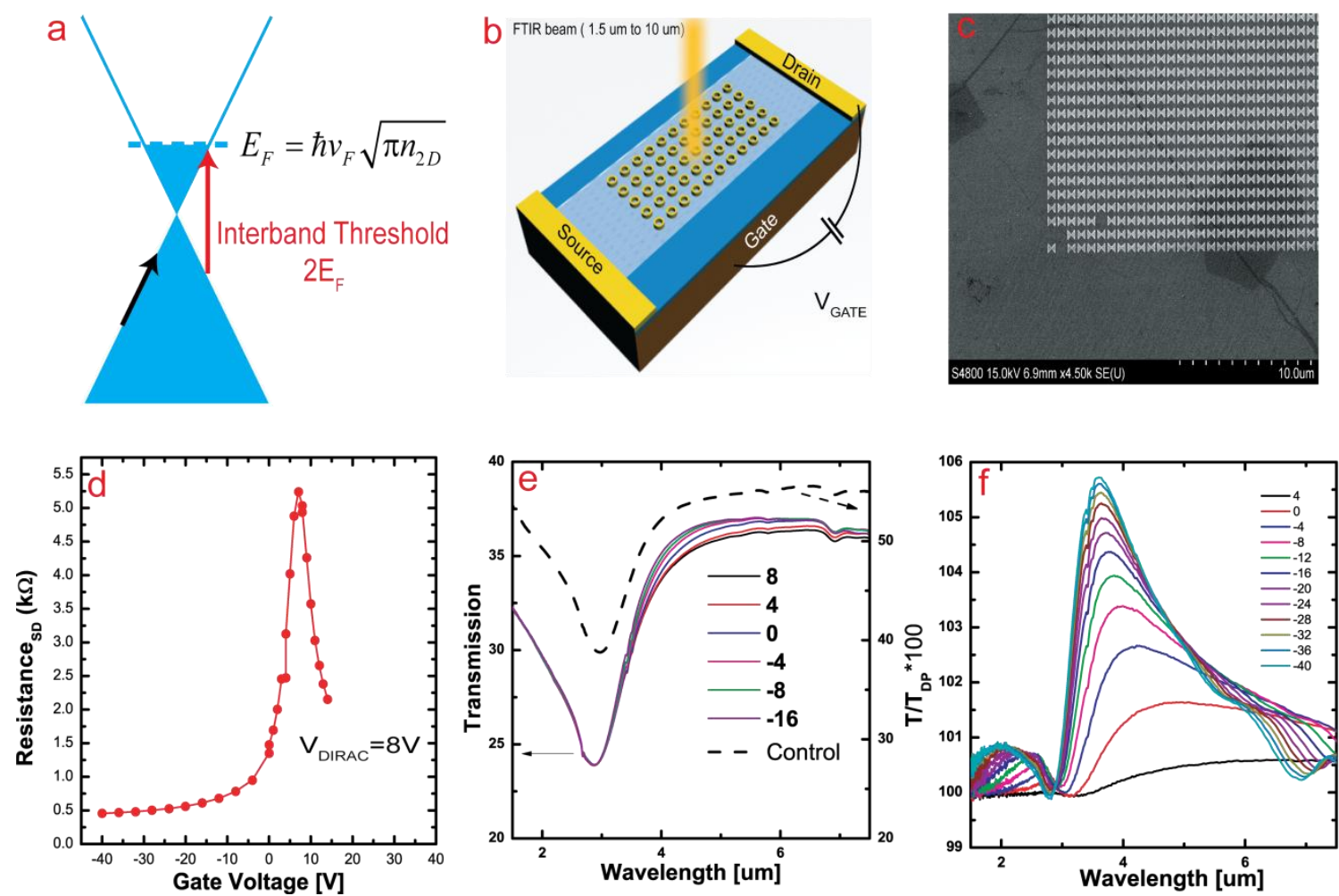

Fig 1. a) Band structure of graphene showing threshold for interband transitions which can be controlled by changing the carrier concentration; b) Schematic of the experimental setup for voltage controlled optical transmission measurements; c) SEM image of the fabricated sample showing the metal nanostructures on top of large area CVD grown graphene; d) In-situ electrical measurements showing carrier concentration change under applied voltage bias; e) Optical transmission measurements of bow-tie structures showing significant change in amplitude with applied voltage.; f) Percentage change in transmission showing peak change at $3.6 \mu \mathrm{m}$ at $40 \mathrm{~V}$ bias.

Acknowledgements: This work was supported in part by ONR MURI on "Large-Area 3D Optical Metamaterials with Tunability and Low Loss" and ARO grant W911NF-11-1-0359.

\section{References}

1. S. Lal, S. Link, and N. J. Halas, "Nano-optics from sensing to waveguiding," Nature Photonics 1, 641-648 (2007).

2. H. T. Chen, W. J. Padilla, J. M. O. Zide, A. C. Gossard, A. J. Taylor, and R. D. Averitt, "Active terahertz metamaterial devices," Nature 444, 597-600 (2006).

3. A. K. Geim and K. S. Novoselov, "The rise of graphene," Nature materials 6, 183-191 (2007).

4. F. Bonaccorso, Z. Sun, T. Hasan, and A. Ferrari, "Graphene photonics and optoelectronics," Nature Photonics 4, 611-622 (2010).

5. M. Jablan, H. Buljan, and M. Soljačić, "Plasmonics in graphene at infrared frequencies," Physical Review B 80, 245435 (2009).

6. F. Koppens, D. Chang, and J. Garcia de Abajo, "Graphene plasmonics: A platform for strong light-matter interactions," Nano letters (2011).

7. A. Vakil and N. Engheta, "Transformation Optics Using Graphene," Science 332, 1291 (2011).

8. M. Liu, X. Yin, E. Ulin-Avila, B. Geng, T. Zentgraf, L. Ju, F. Wang, and X. Zhang, "A graphene-based broadband optical modulator," Nature 474, 64-67 (2011). 